\title{
Failed AtTempt to Induce Chirality Using a Magnetic Field: The Case OF CHIRAL HELICITY OF TRIS-(2-METHYLBENZIMIDAZOL-1-YL)METHANE

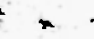 Jose Elguero, ${ }^{*}$ NAdine Jagerovic, ANDreas Werner, AND M." LuISA Jimeno

\author{
Instituto de Química Médica, CSIC, Juan de la Cierva, 3, 28006 Madrid, Spain.
}

\section{ABSTRACT}

Using PTC conditions, the title compound was prepared outside and inside the probe of an NMR spectrometer (7.05 T). In both cases, the racemic mixture was obtained: no enantioselectivity was found.

Recently, Breitmaier and coworkers described enantioselective reactions in a static magnetic field (1). They use, as a model, the transformation of carbonyl compounds (aldehydes and ketones) into carbinols using Grignard reagents and complex metal hydrides. We decided to test this method in a quite different reaction. For this purpose, we selected the synthesis of tris-(2-methylbenzimidazol-1-yl)methane 3 from 2 methylbenzimidazole (2MeBz) 1 and chloroform 2 under PTC conditions (2).
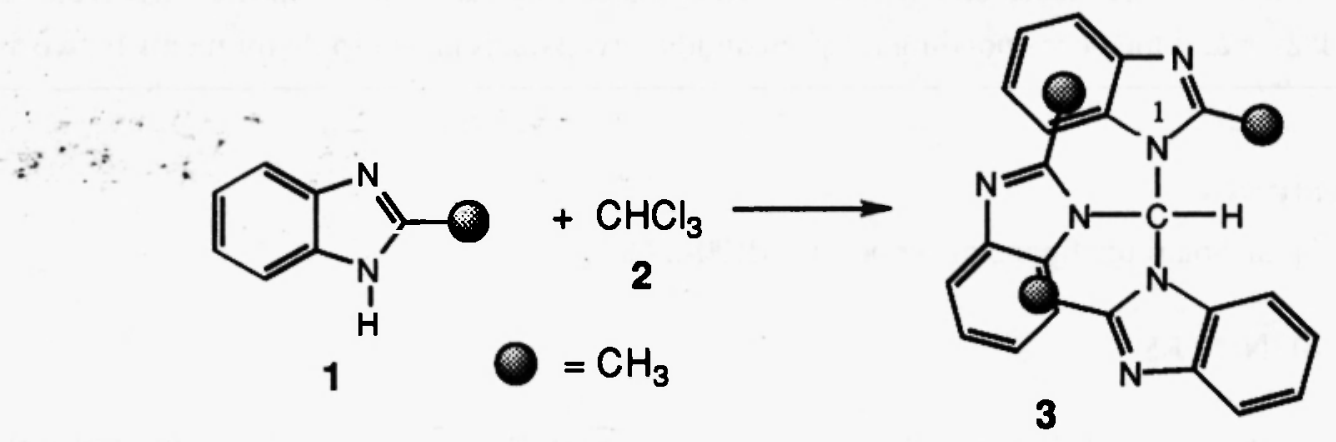

The reaction leads to a racemic compound 3 which was resolved by chromatography on microcrystalline cellulose triacetate. The optical purity was checked by NMR in the presence of Pirkle's alcohol and the racemization barrier was determined: $t_{1 / 2}(\min )=1157 \pm 2\left(D G^{\#}{ }_{343}=28.47 \pm 0.04 \mathrm{kcal} \mathrm{mol}^{-1}\right)$. The two enantiomers correspond to two different "propeller" dispositions related to sterically hindered rotations of the benzimidazole rings about the $\mathrm{N}(1)-\mathrm{C}\left(\mathrm{sp}^{3}\right)$ bonds (3).

We found this example very convenient to test Breitmaier method since the reaction takes place at room temperature in periods of time from minutes to hours (depending on the amount of PTC catalyst) (4) allowing to carry out the reaction in an NMR tube. At the same time, the reaction is quite different from those described (1) with regard of the type of chirality involved.

We first repeated the reaction in the laboratory and obtain the 50:50 mixture of enantiomers as shown by the spectrum in the presence of Pirkle's alcohol (Fig. 1) using the signal of the 2-methyl group. Then, we carried out twice the reaction inside the insert of an NMR spectrometer (Varian XL-300 working at $7.05 \mathrm{~T}$ ), the first time using chloroform and isolating the compound 3 and the second time using deuteriochloroform and 
monitoring the reaction with the spectrometer (formation of $\left.\left[{ }^{2} \mathrm{H}_{1}\right]-3\right)(5)$. In both cases the resulting compound was a 50:50 mixture of both enantiomers (Fig. 1).
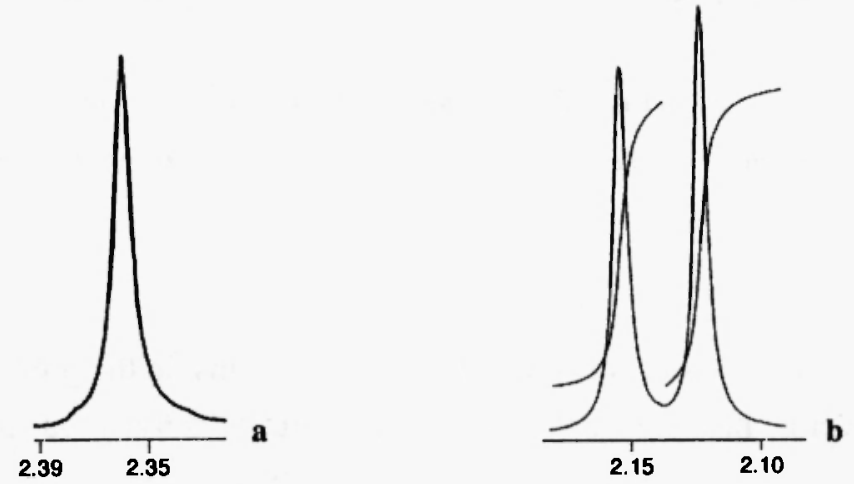

Fig. 1. a) In the absence of Pirkle's alcohol; b) in the presence of Pirkle's alcohol the values are in ppm. (reaction carried out inside the NMR spectrometer)

Compound 3 results from replacing succesively three chlorine atoms by 2 -methyl-benzimidazolyl residues, thus both enantiomers are formed in the last step since for intermediate compounds $(2 \mathrm{MeBz}) \mathrm{CCl}_{2} \mathrm{H}$ and $(2 \mathrm{MeBz})_{2} \mathrm{CClH}$ the racemization barriers should be quite low. In conclusion, it appears that for this particular process there is no enantioselectivity. This may be related to polarity of the species involved although 1-methyl-benzimidazole and the corresponding polybenzimidazolylalkanes have dipole moments between 3 and $4 \mathrm{D}(6)$.

\section{ACKNOWLEDGEMENTS.}

We thank the CICYT of Spain for financial support (FAR90-0746).

\section{REFERENCES AND NOTES}

(1) G. Zadel, C. Eisenbraum, G.J. Wolff and E. Breitmaier, Angew. Chem. Int. Ed. Engl. 33, 454 (1994).

(2) C. Foces-Foces, F.H. Cano, M. Martínez-Ripoll, R. Faure, C. Roussel, R.M. Claramunt, C. López, D. Sanz and J. Elguero, Tetrahedron Asymmetry 1, 65 (1990).

(3) K. Mislow, Acc. Chem. Res. 9, 26 (1976).

(4) V. Bobosik, C. López, R.M. Claramunt, C. Roussel, J.L. Stein, D. Thiery and J. Elguero, Heterocycles 35, 1067 (1993).

(5) Procedure: 2-Methylbenzimidazole ( $0.2 \mathrm{mmole})$, tetrabutylammonium bromide $(0.03$ mmole), potassium hydroxyde $(0.1 \mathrm{mmole})$ and deuteriochloroform $(1 \mathrm{~mL})$ were poured in an NMR tube (10 mm diameter). This tube was then placed in the most intense part of the magnetic field of the spectrometer for $17 \mathrm{~h}$. The evolution of the reaction was followed by ${ }^{13} \mathrm{C} \mathrm{NMR}$. The signal of the methyl carbon of 3 at $15.06 \mathrm{ppm}$ increased while the signal of the methyl carbon of 1 at $14.76 \mathrm{ppm}$ decreased. When no changes in intensity were observed, the ${ }^{1} \mathrm{H}$ NMR spectrum was measured after adding Pirkle's alcohol.

(6) S.B. Bulgarevich, M.D. Grunfest, D.Ya. Movshovich, V. Bobosik, R.M. Claramunt, C. López and J. Elguero, J. Mol. Struct. 274, 197 (1992).

Received June 30, 1994 